Thus the delayed response to the sodium chloride infusion occurred only when the sodium balance of sodium-depleted sheep was significantly altered. Analysis of blood taken from the transverse facial artery of anæsthetized sheep has shown that the sodium concentration rises by 7-35 m.equiv./l. during the infusion of $4 M$ sodium chloride at these rates into the corresponding carotid artery. These considerations indicate that the return to a normal value of the ratio sodium/potassium in the parotid saliva was not due to a direct effect of the increased sodium ion concentration in the blood perfusing the gland. It seems likely that the site of action of the infused sodium ions was elsewhere in the body, and the time delay suggests that either a specific humoral agent was elaborated in response to the infusion, or the production of some agent necessary for the maintenance of a low salivary sodium/potassium ratio was inhibited. Investigation is proceeding to determine the site of the responsive region in the body, and the specific way in which changes in sodium balance may influence it. This experimental procedure, using a conscious undisturbed animal, should be of value in studying the biological regula tion of sodium, since a change of sodium balance induces in the sensitive tissue a response of large magnitude on a short time-scale.

This work was aided by grants from the National Health and Medical Research Council and the Wool Industry Fund of the Commonwealth of Australia. I. R. McDonaLI

D. A. Denton

Department of Physiology,

University of Melbourne. Jan. 3.

1 Denton, D. A., J. Physiol. (in the press).

${ }^{2}$ Verney, F. B., and Vogt, M., Quart. J. Exp. Physiol., 28, 253 (1938).

${ }^{3}$ Verney, E. B., Proc. Roy. Soc. B, 135. 25 (1947).

\section{Effect of Cadmium Salts on Testicular Tissue}

THE physiological action of cadmium salts has been studied extensively by many authors. Morphological disturbances have been described in various organs (liver, kidney, etc.). While studying the effect of cadmium salts on protein metabolism one of us (J. P.) observed that cadmium chloride administered subcutaneously to rats produces macroseopic lesions of the testes. This seems to have escaped the notice of previous authors and therefore this phenomenon was studied more closely.

Mature male rats weighing on an average $300 \mathrm{gm}$. were given $1 \mathrm{mI}$. $0.03 M$ aqueous solution of cadmium chloride or lactate subcutaneously between the shoulder-blades. Male rats injected with an equimolar solution of sublimate served as controls together with normal animals. Rats were killed $2,4,6,8,10$, 12,24 and $48 \mathrm{hr}$. after injection. Already 2-4 hr. after injection of cadmium chloride, capillary stasis and nedema of the interstitium could bo observed microscopically. $8 \mathrm{hr}$. after injection extensive hæmorrhages developed. Occasionally loucocyto infiltration, which was rarely massive, occurred. Regrossive changes of spermiogenic epithelium developed 4-6 hr. after the injection of cadmium chloride (desquamation of cells, pyenosis of nuclei, karyorrhexis and lysis of nuclei). 24-48 hr. after injection all testicular tissue was severely damaged.

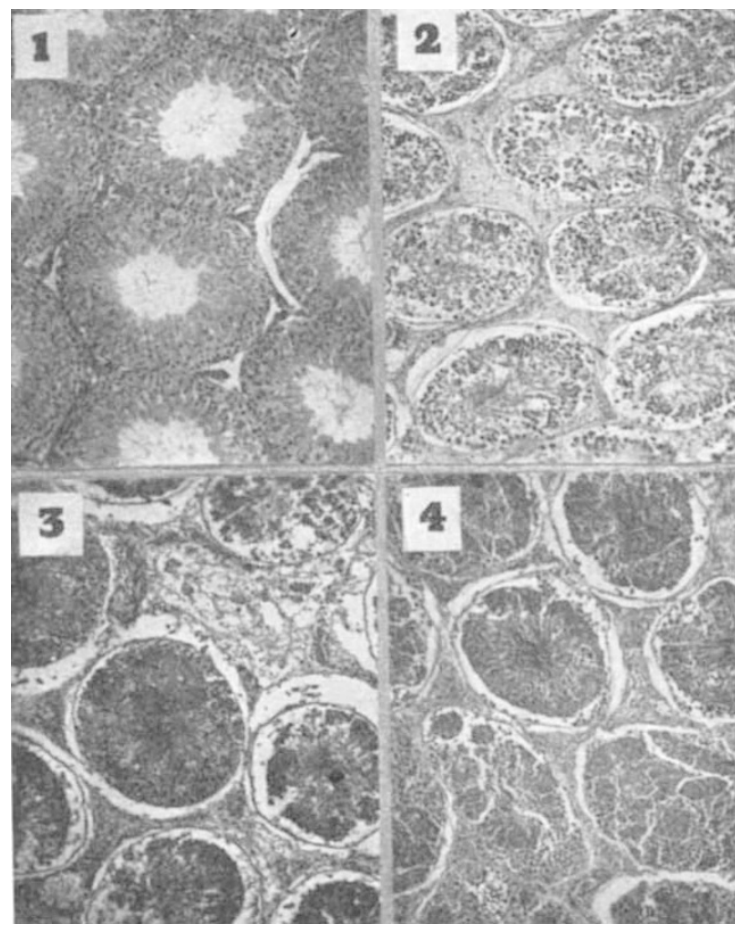

Fig. 1. Sublimate $48 \mathrm{hr}$ after application. That testis of normal appearance. Fig. 2. Cadmium chloride, $48 \mathrm{hr}$. after application. Collapse of tubules, œdema of intertubular spaces, desquamation and pronounced degenerative changes of the spermiogenic epithelium. Fig. 3. Cadmium lactate, $48 \mathrm{hr}$. after application. Histological picture as in Fig. 2. Fig. 4. Cadmium chloride, (Bouin, hæmatoxylin-eosin; $\times$ c. 60 )

On the following days all nuclear remains disappeared and complete testicular necrosis set in. Cadmium chloride and lactate have the same effect, whereas sublimate does not produce any obvious histological changes in the testes. Morphological lesions in the ovaries of female rats after cadmium chloride administration were not observed; but functional changes cannot be excluded. The changes described were found in the testes of all animals tested ( 80 males), whereas changes in other organs were only slight, except in the liver, where they varied in extent.

Work on this problem is being continued.

Czechoslovak Academy of Sciences,

\section{J. Paйízek \\ Z. ZÁHOß̌}

Laboratory of Physiology and

Pathophysiology of Metabolism, Prague, and

II. Institute of Pathology, Charles University,

Prague.

Oct. 25.

IT has been shown (preceding communication) that the subcutaneous application of small amounts of cadmium salts results in a rapid destruction of all tissues of the testicle.

Recently, Elcoate, Fischer, Mawson and Millar showed that prolonged nutritional deficiency of zinc causes testicular injury ${ }^{1}$. It may be asked whether 